\title{
Designing for People who do not Read Easily
}

\author{
Caroline Jarrett \\ Effortmark Ltd \\ 16 Heath Road \\ Leighton Buzzard \\ +441525 370379 \\ Caroline.jarrett@ \\ effortmark.co.uk
}

\author{
Katie Grant \\ Raincharm \\ Communications Ltd \\ 22 Icen Way \\ Dorchester \\ Dorset \\ +441305266651 \\ katie@ \\ raincharm.co.uk
}

\author{
William Wong \\ Interaction Design \\ Centre \\ School of Computing \\ Science \\ Middlesex University \\ +442084112684 \\ w.wong@ \\ mdx.ac.uk
}

\author{
Neesha \\ Kodagoda \\ Interaction Design \\ Centre \\ School of Computing \\ Science \\ Middlesex University \\ +442084112684 \\ neesha.kodagoda@ \\ gmail.com
}

\begin{abstract}
Many people do not read easily for all sorts of reasons: social and cultural, because of impairments, or because of their context. Even in the area of impairments, design for people with learning disabilities might be very different from design for people with visual impairments. But many sets of guidelines, such as WCAG 2.0, are promulgated that attempt to provide one unified approach to design. This workshop will attempt to explore issues in design for people who do not read easily: what do we know, what commonalities can we exploit, and what we need to find out.
\end{abstract}

\section{General Terms}

Human Factors

\section{Keywords}

Design; readability; literacy; accessibility; visual impairments; social access to literacy; neurodiversity; cognitive impairment; learning disability

\section{INTRODUCTION}

Reading is a skill many of us take for granted. We learn at school, practice as adolescents and perfect (or so we hope) the ability as adults. It is something many of us do not even consider as a conscious activity. For many people this is not the case. In addition to reading the text itself, there are often other barriers or obstructions to reading, often as a result of poor design, layout and use of language. This workshop introduces several ideas and approaches to understanding why this should be and will seek to find some solutions to improving the readability of information. In addition to exploring the reasons why some people do not read easily, the workshop will actively encourage attendees to question their own approach to reading and how adaptations/adjustments can be made to improve accessibility for all.

\section{THE PROBLEM SPACE}

We want to explore two key aspects of designing for people who do not read easily:

1. The reasons why reading might be difficult. We propose three categories, and wish to explore whether others find those categories helpful or not.

2. The ways in which we can design for the diversity of this audience.

\subsection{A categorisation of problems?}

People do not read well for many reasons, including (but not limited to) these three categories:

- Social and cultural

- Impairment

- Context

\subsection{The design challenge}

This diverse set of problems affects reading in different ways, for different reasons, but may also overlap. Consider Jason, who has dyslexia. His family didn't read much, there were no books in the house, and the school in his area didn't help either. Now he is trying to make sense of a complex government form in small print that looks frightening. Which of his problems is most important? Are they distinct or not?

And consider Shali: education wasn't available in her village years ago. Now her sight is going a bit. Is designing for her anything like designing for Jason?

And consider Stephen: his profound learning disabilities result from cerebral palsy. He communicates primarily through his helper. Is designing for him anything like designing for Shali or Jason?

It seems rather likely that these are separate challenges, but we also know, for example, that people with visual impairments do not want to have a separate web site just for them: they want to be able to experience the same web site as everyone else, albeit in a different way.

\subsection{A common set of guidelines?}

When it comes to designing for Jason, Shali, and the many other people who do not read easily, what do we know and what are we researching? Are we asking the right questions or are we making assumptions about what we believe someone might need. Fundamentally, are we asking at all?

Whilst guidelines may have been written, have they been tested and evaluated with the audiences they are aimed at? The problem set is wide - how do we capture and engage with these 
people? How do we consider their needs? Also, by engaging in consultation/user testing etc there is then an expectation to deliver and improve provision. Where does this support and commitment come from? Whose responsibility is it?

\section{The workshop objectives}

This workshop aims to be initial and exploratory. We want to bring together people working on any aspect of the problem of designing for people who do not read easily, including (but not limited to) the reasons why reading might be difficult, the ways in which we can design to make reading easier, sets of guidelines to improve design.

One question we will consider is where does design end and assistive technology begin? Should we be designing so that specific technologies can help people to read easily? Or designing so that assistive technologies are not necessary? What is classed as inaccessible design?

In this workshop we plan to focus primarily on the design. As an example of one design technique, we have attached below version of this description written in easier langauge.

Our initial aim is to collect and publish resources on our new Wiki [url TBA]. We wish to foster both formal and informal contacts between the many people who are working on different aspects of this field.

We see this as the first formal event in a series that will start with email and Wiki discussions, together with face-to-face informal special interest gatherings at two key USA conferences in June (STC and UPA). Our hope is that it will become the foundation of further events at other major conferences, with the eventual aim of producing a publication.

\section{THE CONFERENCE OBJECTIVES}

What is 'culture, creativity and interaction' if the simple barrier of not being able to read easily excludes parts of our audiences? How can we help people to cross cultural barriers, such as social group or language? How can we ensure that our designers are as creative as they should be, while still making their work accessible to all?

\section{OUTLINE OF THE DAY}

We will cover:

- Definitions

- Terminology

- 'Try it' session

- 'Best of the best' session

- Testing with your audience

\section{REFERENCES}

[1] Jarrett, C., Quesenbery, W, and Roddis, I. Applying usability principles to content for diverse audiences HCI 2006

\section{Making things easier to read: What this workshop is about}

\section{Workshop}

A workshop is a place for people to meet and share their ideas.

We want to meet to talk about how to make books and pages easy to read and to understand. It is very important that everyone has the chance to read.

Here are some of the people who might have problems with reading:

- People who have not had a chance to learn as children.

- People who cannot see well.

- People who have a learning disability.

- People who have autism.

- People who have dyslexia.

- People who need to read when the light is bad.

- People who need to read when they are upset or tired.

Autism

People who have autism can find it difficult to communicate and be with other people.

\section{Dyslexia}

People who have dyslexia can find it hard to read and write. They can get words and letters muddled up.

Sometimes the way that words and pictures are put together can make things hard to understand. We want to invite people to talk about this in our workshop.

We want to write some rules for people to follow to make the words and pictures easier to read. There might need to be different rules for different groups.

If the rules are followed then it will make things easier to read for everyone.

This work will not be for one day only. We want the work we do to continue.

We will test our results and write them down.

We will put our results on our web site. We will publish the address later.

You can find out more about our work if you go to our web site. 